\title{
Unicité et stabilité de l'équilibre dans une économie de production avec règle de tarification marginale : les cas convexe et non-convexe
}

\section{Elyès JOUINI*}

RÉSUMÉ. - Dans cet article nous établissons une formule de l'indice pour une économie où les producteurs utilisent la tarification marginale et nous montrons que, sous des hypothèses de monotonicité sur la correspondance d'excès de demande et les conditions suffisantes du second ordre de maximisation du profit, l'économie considérée admet un unique équilibre. Les résultats anisi obtenus étendent les conditions d'unicité connues dans le cas de l'équilibre compétitif et les généralisent dans le cas d'ensembles de production non-convexes.

\section{Uniqueness and Stability of the Marginal Pricing} Equilibrium: The Convex and Nonconvex Cases

ABSTRACT. - In this paper we establish an index formula for a marginal pricing production economy and we prove the uniqueness of the equilibrium under a monotonicity assumption on the excess demand function and the local second order sufficient conditions for profit maximization. Our result generalizes the known results in the competititve framework and extends them to economies with possibly nonconvex production sets.

\footnotetext{
* E. J OUINI: ENSAE.
} 


\section{Introduction}

L'unicité et la stabilité de l'équilibre dans une économie d'échange sont généralement étudiés sous des hypothèses de monotonicité (hypothèse de substituabilité brute, hypothèse des préférences révélées...) sur la fonction de demande globale. Ces résultats peuvent être généralisés à l'équilibre compétitif d'une économie avec production sous des hypothèses additionnelles assez faibles sur les ensembles de production (voir KEHOE [1983] et MAS-ColeLL [1985]). Cependant, l'hypothèse de monotonicité introduite par ces derniers porte sur une fonction de demande dont les variations dépendent aussi bien du secteur de consommation que du mode de tarification du secteur productif. De plus, ces deux dépendances sont fortement imbriquées ce qui laisse peu de place à l'interprétation de cette hypothèse en terme de fondamentaux de l'économie étudiée.

Par ailleurs, la littérature s'intéressant au problème des rendements croissants dans les ensembles de production s'est attaché à analyser un environnement économique dans lequel les producteurs sont modélisés par des ensembles de productions non-convexes et des règles de tarification générales. Parmi ces règles de tarification, la règle de tarification marginale a toujours suscité un intérêt particulier en raison de ses propriétés relatives à l'optimalité paretienne, comme l'a montré GUESNERIE [1975], avec un formalisme mathématique légèrement différent de celui que nous adopterons. Cepedant, cette littérature comporte peu de contributions relatives à l'unicité de l'équilibre. En effet, les principaux résultats en la matière sont obtenus comme conséquence d'une formule de l'indice qui permet d'énoncer des conditions mathématiques d'unicité (voir KAMIYA [1988] et JouINI [1992]). Il est à noter que cette dernière référence tente d'apporter une interprétation économique aux conditions mathématiques d'unicité mais le modèle considéré ne prend en compte que les tarifications à pertes bornées et ignore donc, en général la règle de tarification marginale. En outre, les hypothèses qui y sont faites concernant la fonction de demande globales sont de la même nature que celles de KeHOE [1983] et MAS-COLELL [1985] et donc peu satisfaisantes. De plus, BonnisSEAU et CORNET [1988] ont montré que la règle de tarification marginale est à pertes bornées si et seulement si l'ensemble de production est étoilé ce qui n'est pas le cas d'un grand nombre d'exemples élémentaires. Ainsi, ni les ensembles de production définis par des fonctions de production CES à rendements croissants, ni l'ensemble de production intervenant dans le fameux exemple de BEATO et MAS-COLELL [1985] ne sont étoilés. Par ailleurs, contrairement à ce que pourrait suggérer l'intuition, la tarification marginale ne peut pas être modifiée « à l'infini » de telle façon à obtenir une tarification à pertes bornées. Toutefois, le résultat allant le plus loin dans ce sens est dû à BoNNISSEAU [1992] et montre qu'il existe toujours une économie avec règles de tarifications à pertes bornées ayant le même ensemble d'équilibres qu'une économie de tarification marginale donnée.

Ainsi, jusqu'à cet article de BonNisSEAu [1992], les règles de tarification marginale et à pertes bornées ont donné lieu à deux littératures séparées utilisant pour la démonstration de l'existence de l'équilibre des techniques différentes. 
Dans cet article, nous utilisons ce résultat récent de BoNNISSEAU [1992] pour généraliser les résultats de JouinI [1992] et établir une formule de l'indice pour des économies de tarification marginale et pour lesquels les pertes ne sont pas nécessairement bornées. Nous montrons alors que sous les hypothèses classiques de monotonicité sur la correspondance d'excès de demande et sous les conditions suffisantes du second ordre de maximisation du profit ainsi qu'une condition technique de transversalité, une telle économie admet un unique équilibre. A la différence des articles précédemment cités, notre correspondance de demande est définie de telle sorte que ses variations soient indépendantes du secteur productif et égales à ce qu'elle serait dans l'économie d'échange que l'on pourrait naturellement associer à l'économie étudiée. Nous montrons, de plus, que la condition technique imposée est notamment satisfaite sous les conditions suffisantes du second ordre de maximisation du profit ou dans le cas d'un ensemble de production défini par une matrice d'activité et tel que à l'équilibre il $\mathrm{y}$ a toujours autant d'activités qui opèrent que le nombre total de biens moins un (condition de SAMUELSON [1951]). Ainsi, lorsque les ensembles de production sont différentiellement strictement convexes (i.e. lorsqu'ils satisfont les conditions suffisantes du second ordre de stricte convexité), ou lorsqu'ils sont somme d'un tel ensemble et d'une partie représentant un coût fixe et tels que, à l'équilibre tous les producteurs sont "productifs », alors l'unicité de l'équilibre est assurée.

Ainsi, il nous semble que l'intérêt essentiel de cet article est de montrer

- d'une part, que dans le cas d'ensemble de productions convexes, l'unicité de l'équilibre est obtenue sous les mêmes conditions que pour les économies d'échange ou presque, il suffit que les ensembles soient différentiellement strictement convexes, et donc que l'unicité de l'équilibre compétitif est une condition moins " évasive » (en angl. elusive) que ne l'affirme KeHOE [1983]) dans sa conclusion,

- d'autre part, que dans le cas général, l'unicité est directement liée à des situations d'équilibres non localement efficiente pour l'un des producteurs (possibilité d'augmenter son profit par une modification marginale de la production).

Dans les parties 2 et 3 de cet article, nous décrivons le cadre général de notre étude et nous établissons la formule de l'indice annoncée, cette dernière étant démontrée en appendice. Un résultat d'existence d'un équilibre pour une économie avec producteurs utilisant la règle de tarification marginale s'en déduit alors. En partie 4, nous établissons des résultats d'unicité et de stabilité concernant cet équilibre.

Rappelons à présent les quelques définitions suivantes. Pour un ensemble fermé $Y \in R^{\ell}$, un vecteur perpendiculaire à $Y$ en $y \in Y$ est un élément de l'ensemble:

$$
\perp_{Y}(y)=\left\{p \in R^{\ell}: \exists \rho \in R, \forall y^{\prime} \in Y, p \cdot y \geq p \cdot y^{\prime}-\rho\left\|y-y^{\prime}\right\|^{2}\right\} .
$$

Le cône normal de Clarke à $Y$ en $y$ (voir Clarke [1983]), noté $N_{Y}(y)$ est alors l'enveloppe convexe fermée de l'ensemble:

$\left\{p \in R^{\ell}: \exists\left(y_{q}\right) \subset Y,\left(y_{q}\right) \rightarrow y, \exists\left(p_{q}\right) \subset R^{\ell},\left(p_{q}\right) \rightarrow\right.$ pet $\left.\forall q, p_{q} \in \perp_{Y_{q}}(y)\right\}$. 


\section{Le modèle ${ }^{1}$}

Soit $\mathcal{E}$ une économie comportant $\ell$ biens, $(h=1, \ldots, \ell), m$ consommateurs, $(i=1, \ldots, m)$, et $n$ firmes, $(j=1, \ldots, n)$, et soit $\omega=$ $\left(\omega_{1}, \ldots, \omega_{m}\right) \in R^{\ell m}$ le vecteur des allocations initiales des consommateurs. La technologie de la $j$-ième firme est décrite par $Y_{j} \subset R^{\ell}$, et nous supposons que les choix des producteurs satisfont aux conditions du premier ordre de maximisation du profit. Plus précisément, pour tout plan de production efficient, $y_{j} \in \partial Y_{j}$, la firme choisit $p$ dans $\varphi_{j}\left(y_{j}\right) \stackrel{\text { def }}{=} N_{Y_{j}}\left(y_{j}\right) \cap S$, où $N_{Y_{j}}\left(y_{j}\right)$ est le cône normal de Clarke à $Y_{j}$ en $y_{j}$ et $S=\left\{p \in R_{+}^{\ell}: \sum_{h=1}^{\ell} p_{h}=1\right\}$ est l'ensemble des prix normalisés. La correspondance ${ }^{2} \varphi_{j}\left(y_{j}\right)$ est appelée règle de tarification marginale ${ }^{3}$.

Le comportement du $i$-ième consommateur est décrit par la correspondance $D_{i}: S_{++} \times R_{++} \rightarrow R_{+}^{\ell}$, (où $S_{++}=S \cap R_{++}^{\ell}$ est l'intérieur relatif de $S$ ), qui à un vecteur de prix $p \in S_{++}$et un revenu strictement positif $w_{i}$ associe l'ensemble $D_{i}\left(p, w_{i}\right)$ des plans de consommation possibles pour le $i$-ième consommateur. Le revenu $w_{i}$ de ce consommateur est défini, pour des plans de productions $\left(y_{j}\right)$ et un vecteur de prix $p$, par $w_{i}=p \cdot w_{i}+r_{i}$ $\left(p ; p \cdot y_{1}, \ldots, p \cdot y_{n}\right)$ où $r_{i}$ représente la règle de distribution des revenus des différentes firmes entre les agents. Plus précisément, $\left(r_{i}\right)$ est une fonction continue à valeurs réelles définie pour toute famille prix-profits $\left(p ; \pi_{1}, \ldots, \pi_{n}\right)$ dans $S \times R^{n}$ telle que $\sum_{i=1}^{m} r_{i}\left(p ; \pi_{1}, \ldots, \pi_{n}\right)=\sum_{j=1}^{n} \pi_{j}$.

Nous pouvons introduire à présent les hypothèses principales de notre modèle:

Hypothèse (A) :

- A-1 pour tout $i, D_{i}$ est une correspondance semi-continue supérieurement à valeurs convexes compactes non-vides dans $R_{+}^{\ell}$, vérifiant la loi de Walras (i.e. pour tout $(p, w)$ dans $S_{++} \times R_{++}$et tout $x$ dans $D_{i}(p, w)$ on a $\left.p \cdot x=w\right)$, et telle que pour toute suite $\left(p^{q}, w^{q}\right)$ dans $S_{++} \times R_{++}$convergeant vers $(p, w)$ avec $w>0$ et $p \notin S_{++}$on a $d\left(0, D_{i}\left(p^{q}, w^{q}\right)\right) \rightarrow+\infty$.

1. Soient $x=\left(x_{h}\right), y=\left(y_{h}\right)$ des vecteurs de $R^{\ell}$, on note $x \cdot y=\sum_{h=1}^{l} x_{h} y_{h}$ le produit scalaire de $R^{\ell}$, et $\|x\|=(x \cdot x)^{\frac{1}{2}}$ la norme euclidienne. On note $x \geq y$ (resp. $x \gg y$ ) pour $x_{h} \geq y_{h}$ (resp. $x_{h}>y_{h}$ ) pour tout $h$, on note alors $R_{+}^{\ell}=\left\{x \in R^{\ell}: x \geq 0\right\}$ et $R_{++}^{\ell}=\left\{x \in R^{\ell}: x \gg 0\right\}$. On note $e$, le vecteur de $R^{\ell}$ dont toutes les coordonnées sont égales à 1 . Pour $A \subset R^{\ell}$, on note $\partial A$ la frontière de $A$ et si $A$ est un sous-espace de $R^{\ell}$ on note $\operatorname{proj}_{A}$ la projection orthogonale sur $A$.

2. Soient deux espaces topologiques $X$ et $Y$, une correspondance $\phi$ à valeurs convexes compactes non-vides est dite semi-continue supérieurement si elle est localement bornée et si son graphe, i.e. $\{(x, y) \in X \times Y: y \in \phi(x)\}$, est fermé.

3. Rappelons que $\varphi_{j}$ est une correspondance semi-continue supérieurement à valeurs convexes compactes non-vides qui dans le cas des ensembles de production convexes s'identifie avec la règle de maximisation du profit. 
- A-2 pour tout $j, Y_{j}$ est un ensemble fermé non-vide vérifiant l'hypothèse de libre disposition, i.e., $Y_{j}-R_{+}^{\ell} \subset Y_{j}$. De plus, pour tout $z$ dans $R^{\ell}$, $\left\{\left(y_{j}\right) \in \prod_{j=1}^{n} Y_{j}: \sum_{j=1}^{n} y_{j} \geq z\right\}$ est borné. ${ }^{4}$

Nous définissons alors un équilibre de tarification marginale de la façon suivante :

DÉFINITION 1 : Un équilibre de tarification marginale de l'économie $\mathcal{E}=\left(\left(D_{i}, r_{i}, \omega_{i}\right)\left(Y_{j}\right)\right)$ est un élément $\left(\left(x_{i}\right),\left(y_{j}\right), p\right)$ de $R^{\ell m} \times \prod_{j=1}^{n} Y_{j} \times$ $S_{++}$tel que

(i) $x_{i} \in D_{i}\left(p, p \cdot \omega_{i}+r_{i}\left(p ; p \cdot y_{1}, \ldots, p \cdot y_{n}\right)\right)$, pour tout $i$,

(ii) $\left(\left(y_{j}\right), p\right)$ est un équilibre de production pour la règle de tarification marginale, i.e. $y_{j} \in \partial Y_{j}$ et $p \in \varphi_{j}\left(y_{j}\right)$, pour tout $j$,

(iii) $\sum_{i=1}^{m} x_{i}=\sum_{j=1}^{n} y_{j}+\sum_{i=1}^{m} \omega_{i}$.

Puisque dans ce qui suit tous les paramètres de l'économie $\mathcal{E}$, sauf éventuellement les allocations initiales $\omega$, seront considérés comme fixés nous noterons $\mathcal{E}_{\omega}$ ou, pour simplifier, $\omega$, cette économie. Dans ce qui suit, et afin d'établir les résultats d'existence et d'unicité d'un équilibre de tarification marginale, nous allons supposer que si $\left(\left(y_{j}\right),\left(\omega_{i}\right)\right)$ est une allocation admissible et si $\left(\left(y_{j}\right), p\right)$ est un équilibre de production alors le revenu global de l'économie est strictement positif. BONNISSEAU et CORNET [1988] et KAMIYA [1988] ont montré que cette hypothèse, dite hypothèse de survivance, est une condition nécessaire d'existence de l'équilibre de tarification marginale.

HYPOTHĖSE $S(\omega)$ : Pour tout $\omega^{\prime} \geq \omega$, pour tout équilibre de production $\left(\left(y_{j}\right), p\right)$ tel que $\sum_{j=1}^{n} y_{j}+\sum_{i=1}^{m} \omega_{i}^{\prime} \geq 0$ on a $p \cdot\left(\sum_{j=1}^{n} y_{j}\right.$ $\left.+\sum_{i=1}^{m} \omega_{i}^{\prime}\right)>0$.

\section{Economies régulières et formule de l'indice}

Dans cette section nous allons étudier la correspondance de demande associée à notre économie ou plus précisément une extension de cette dernière.

Pour celà, considérons l'ensemble $U$ défini par

$U=\left\{\left(\left(y_{j}\right), p,\left(\omega_{i}\right)\right) \in \prod_{j=1}^{n} \partial Y_{j} \times S_{++} \times R^{\ell m}: p \cdot\left(\sum_{j=1}^{n} y_{j}+\sum_{i=1}^{m} \omega_{i}\right)>0\right\}$,

4. Cette condition est plus faible que la condition classique portant sur le cône asymptotique engendré par l'ensemble de production global $A(Y) \cap-A(Y)=\{0\}$ et dans le cas convexe est plus faible que l'hypothèse d'irréversibilité $Y \cap(-Y)=\{0\}$ (voir Debreu [1959] et HuRwicz, REITER [1973]). 
et soient également les fonctions $\tilde{r}_{i}: U \rightarrow R,(i=1, \ldots, m)$ définies par

$$
\tilde{r}_{i}=(1-\theta(\rho)) \frac{\sum_{i=1}^{m} \rho_{i}}{m}+\theta(\rho) \rho_{i}-p \cdot \omega_{i}
$$

où $\rho=\left(\rho_{i}\right)=\left(p \cdot \omega_{i}+r_{i}\left(p ; p \cdot y_{1}, \ldots, y_{n}\right)\right.$, et où

$$
\theta(\rho)= \begin{cases}1, \quad \text { si pour tout } i, & \rho_{i}>0 \\ \frac{\sum_{i=1}^{m} \rho_{i}}{\sum_{i=1}^{m} \rho_{i}-m \inf _{k} \rho_{k}}, & \text { dans tous les autres cas. }\end{cases}
$$

Il est facile de vérifier que ces fonctions vérifient les conditions (i), (ii) et (iii) suivantes:

(i) pour tout $k=1, \ldots, m$, et pour tout $\left(\left(y_{j}\right), p,\left(\omega_{i}\right)\right) \in U$, $\tilde{r}_{k}\left(\left(y_{j}\right), p,\left(\omega_{i}\right)\right)+p \cdot \omega_{k}>0$

(ii) si pour $\left(\left(y_{j}\right), p,\left(\omega_{i}\right)\right) \in U$ et pour $k \in\{1, \ldots, m\}, p \cdot \omega_{k}+$ $r_{k}\left(p ; p \cdot y_{1}, \ldots, p \cdot y_{n}\right)>0$ alors pour tout $k, r_{k}\left(p ; p \cdot y_{1}, \ldots, p \cdot y_{n}\right)=$ $\tilde{r}_{k}\left(\left(y_{j}\right), p,\left(\omega_{i}\right)\right)$.

(iii) pour tout $\left(\left(y_{j}\right), p,\left(\omega_{i}\right)\right) \in U, \sum_{i=1}^{m} \tilde{r}_{i}\left(\left(y_{j}\right), p,\left(\omega_{i}\right)\right)=p \cdot \sum_{j=1}^{n} y_{j}$.

Soit, à présent, la correspondance $\Lambda_{0}$ à valeur dans $\left(e^{\perp}\right)^{n+1} \times R^{\ell m}$ et définie sur $U$ par $\Lambda_{0}\left(\left(y_{j}\right), p,\left(\omega_{i}\right)\right)=\prod_{j=1}^{n}\left(\varphi_{j}\left(y_{j}\right)-\{p\}\right) \times$ $\bar{F}\left(\left(y_{j}\right), p,\left(\omega_{i}\right)\right) \times\left\{\left(\omega_{i}\right)\right\}$, où $F\left(\left(y_{j}\right), p,\left(\omega_{i}\right)\right)=\sum_{i=1}^{m} D_{i}\left(p, p \cdot \omega_{i}+\right.$ $\tilde{r}_{i}\left(\left(y_{j}\right), p,\left(\omega_{i}\right)\right)-\sum_{j=1}^{n} y_{j}-\sum_{i=1}^{m} \omega_{i}$, et où, pour $z$ dans $R^{\ell}, \bar{z}$, désigne la projection orthogonale de $z$ sur $e^{\perp}$.

Il est clair alors que $F$ est égale à la correspondance d'excès de demande dans toutes les situations $\left(\left(y_{j}\right), p,\left(\omega_{i}\right)\right)$ telles que les revenus de tous les consommateurs sont strictement positifs. Cette remarque nous amène à considérer l'hypothèse suivante:

HyPOTHĖSe $\mathrm{R}(\omega)$ : pour tout équilibre de production $\left(\left(y_{j}\right), p\right)$ tel que $p \gg 0$ et $p \cdot\left(\sum_{j=1}^{n} y_{j}+\sum_{i=1}^{m} \omega_{i}\right)>0$ on a, pour tout $i, p \cdot \omega_{i}+r_{i}(p ; p$. $\left.y_{1}, \ldots, p \cdot y_{n}\right)>0$.

Cette dernière hypothèse, complémentaire de l'hypothèse de survivance, assure que pour tout équilibre de production pour lequel le revenu global de l'économie est strictement positif, les richesses individuelles des agents sont strictement positives. Couplées avec l'hypothèse de survivance, on a alors que pour tout équilibre de production admissible, toutes les richesses individuelles sont strictement positives. Ces deux hypothèses n'en sont pas moins, économiquement et mathématiquement, de nature différente. L'hypothèse de survivance porte sur la géométrie de l'ensemble des équilibres de production et donc sur l'interaction entre les différents producteurs imposée par la règle de tarification marginale. L'hypothèse $R(\omega)$, porte quant à elle sur les règles de distribution des revenus, imposant que chaque individu puisse avoir une part strictement positive d'une richesse strictement positive.

Sous l'hypothèse $(\mathrm{S}(\omega))$, il est facile de voir que si $\left(\left(x_{i}\right),\left(y_{i}\right), p\right)$ est un équilibre de tarification marginale de $\mathcal{E}_{\omega}$ alors $\left(\left(y_{j}\right), p,\left(\omega_{i}\right)\right) \in \Lambda_{0}^{-1}(0,0, \omega)$ et que sous l'hypothèse $(\mathrm{R}(\omega))$, la réciproque est également vérifiée. 
Il suffit alors d'étudier la correspondance $\Lambda_{0}$, et plus précisément son degré (pour une définition mathématique du degré voir CELLINA et LASOTA [1969], voir également l'appendice de JouINI [1992]), pour connaître le nombre algébrique d'équilibres de $\mathcal{E}_{\omega}$.

Plus précisément, nous allons introduire les notions d'économie régulière et d'indice d'un équilibre de telle sorte qu'il soit possible de déterminer pour toute économie régulière $\mathcal{E}_{\omega}$, la somme des indices de ses équilibres.

Rappelons que, selon BonNISSEAU et CORNET [1988], la restriction de $\operatorname{proj}_{e^{\perp}}$ (la projection orthogonale sur $e^{\perp}$ ) à $\partial Y_{j}$ est un homéomorphisme pour les ensembles de production $Y_{j}$ vérifiant l'hypothèse (A-2). Par conséquent, pour tout $j$, l'ensemble $\partial Y_{j}$ peut être muni, de façon canonique, d'une structure de variété $C^{1}$. Il faut bien remarquer que cela n'implique pas que $\partial Y_{j}$ est une sous-variété $C^{1}$ de $R^{n}$ (pour la structure induite par l'injection canonique dans $R^{n}$ ), ce qui supposerait un bord lisse, mais seulement que, homéomorphe à une variété $C^{1}, \partial Y_{j}$ peut être muni d'une structure de variété abstraite de même nature. Ainsi, dans toute la suite la paramétrisation de $\partial Y_{j}$ considérée sera celle associée à la projection de $\partial Y_{j}$ sur $e^{\perp}$. Ce qui signifie que si $y_{j}$ continue à être un vecteur de $R^{n}$, dans toute la suite $d y_{j}$ est intuitivement un vecteur infinitésimal de $e^{\perp}$.

DÉfINITION 2: L'éconimie $\mathcal{E}_{\omega}$, ou pour simplifier $\omega$, est régulière si (i) $\Lambda_{0}$ est $C^{1}$ dans un voisinage de $\Lambda_{0}^{-1}(0,0, \omega)$,

(ii) $(0,0, \omega)$ est une valeur régulière de $\Lambda_{0}$.

En particulier, si $\omega$ est régulière alors $\Lambda_{0}$, les règles de tarification et la correspondance de demande sont uni-valuées dans un voisinage des équilibres de $\omega$.

DÉfINITION 3 : Si $\mathcal{E}_{\omega}$ est une économie régulière et si $\left(\left(x_{i}\right),\left(y_{i}\right), p\right)$ est un équilibre de cette économie, alors l'indice de cet équilibre est défini par le signe $(+1$ ou -1$)$ de $\operatorname{det} \Lambda_{0}^{\prime}\left(\left(y_{j}\right), p,\left(\omega_{i}\right)\right)$ et est noté $i_{\left(\left(x_{i}\right),\left(y_{i}\right), p\right)}$.

Nous pouvons à présent établir la formule de l'indice suivante:

THÉORÈME 1 : Soit $\mathcal{E}$ une économie régulière définie par $\left(\left(D_{i}, r_{i}, \omega_{i}\right)\left(Y_{j}\right)\right)$ et vérifiant les hypothèses $(1),(S(\omega))$ et $(R(\omega))$, on a

$$
\sum i_{\left(\left(x_{i}\right),\left(y_{i}\right), p\right)}=(-1)^{\ell-1}
$$

où la somme porte sur tous les équilibres de $\mathcal{E}$.

La preuve de ce résultat sera donnée, en appendice, à la fin de cet article. Cependant, nous pouvons d'ores et déjà énoncer le corrolaire immédiat suivant:

Corollaire 2 : Sous les hypothèses du théorème 1 , l'économie $\mathcal{E}$ admet un nombre impair d'équilibres et donc admet au moins un équilibre. 


\section{Unicité et stabilité de l'équilibre}

Dans la littérature sur les économies d'échanges, l'unicité de l'équilibre est, en général, prouvée sous une condition de monotonicité de la correspondance d'excès de demande (i.e. $\left.\sum_{i} D_{i}\left(p, p \cdot \omega_{i}\right)-\sum_{i} \omega_{i}\right)$ ou plus précisément sur le comportement de cette correspondance au voisinage de l'équilibre. Nous renvoyons à MAS-CoLELL [1985] pour voir que toutes ces hypothèses (substituabilité brute, préférences révélées, ...) sont en fait des conditions suffisantes de négativité du déterminant jacobien de la fonction d'excès de demande au voisinage des prix d'équilibre.

Dans le cas qui nous intéresse, la correspondance d'excès de demande peut être définie par $Z(p)=\sum_{i} D_{i}\left(p, p \cdot \omega_{i}+r_{i}\left(p ; p \cdot y_{1}, \ldots, p \cdot y_{n}\right)-\sum_{i} \omega_{i}-\right.$ $\sum_{j=1}^{n} y_{j}$. Définie de cette façon, les variations de $p$ n'entraînent pas un repositionnement des producteurs. Ainsi, les seules modifications de revenu prises en compte sont celle dues au mouvement des prix et non celles dues au mouvement des plans de production qui en résulte. En celà, la sensibilité de notre fonction d'excès de demande par rapport au prix est identique à celle de la fonction de demande dans l'économie d'échange canoniquement associée à notre économie. Alors que, dans KeHOE [1983] et MAS-COLELL [1985], la fonction d'excès de demande considérée correspondrait à notre fonction $Z$ dans laquelle on remplacerait $y_{j}$ par $\varphi_{j}^{-1}(p)$ partout où celà aurait un sens.

Nous dirons d'une économie qu'elle est « $C^{1} »$, si elle vérifie la condition (i) de la définition de la régularité (définition 2). En d'autres termes, une économie est « $C^{1}$ » si, au voisinage des allocations d'équilibre, les règles de tarifications et la correspondance d'excès de demande sont $C^{1}$. Il est immédiat qu'une économie régulière est " $C^{1}$ » et que la réciproque est a priori fausse. Pour la bonne définition des hypothèses qui vont suivre, nous supposerons que l'économie considérée est « $C^{1} »$. Cette hypothèse sera cependant rappelée dans tous les énoncés de résultats. Nous pouvons donc imposer que

HYPOTHĖSE DE SUBSTITUABILITÉ BRUTE : pour tout équilibre de l'économie $\omega$, pour tout $(h, k) \in\{1, \ldots, \ell\}$ avec $h \neq k, \frac{\partial Z^{k}}{\partial p_{h}}>0$ et $\frac{\partial Z^{k}}{\partial p_{k}}<0$.

En d'autres termes, cette hypothèse signifie qu'un accroissement du prix d'un bien entraîne la diminution de la demande en ce bien et par un effet compensatoire une augmentation de la demande en tous les autres biens. Cette hypothèse, très fréquemment utilisée dans le cadre des économies d'échange, est cependant très restrictive et dès que le nombre de biens est supérieur strictement à deux, il n'y a aucune justification raisonnable à cette hypothèse. C'est pour cette raison que nous serons plutôt amenés à étudier l'hypothèse plus faible suivante, pour laquelle nous nous référons à MAS-COLELL [1985]. La référence à la substituabilité brute étant maintenue pour des raisons historiques et comme référence.

LOI DE DEMANDE GÉNÉRALISÉE : pour tout équilibre $\varepsilon$ de l'économie $\omega$, pour tout $v \in R^{\ell}, v \neq 0$ et $p \cdot v=0$ on a $v \cdot \frac{d Z}{d p} v<0$. 
L'interprétation économique de cette hypothèse est claire. La conclusion $v \cdot \frac{d Z_{\varepsilon}}{d p} v<0$ sous la condition $p \cdot v=0$ signifie, de manière informelle que, à ensemble de budget fixé, la demande varie en sens inverse des prix et ceci tant pour les biens «simples» que pour les paniers de biens («biens composés »). C'est en ce sens qu'elle est qualifiée de loi de la demande généralisée.

Par ailleurs, nous allons supposer que les producteurs vérifient au voisinage des plans de production d'équilibre, non seulement les conditions nécessaires du premier ordre de maximisation du profit (règle de tarification marginale), mais également les conditions nécessaires du second ordre d'une telle maximisation. Il est peut-être utile de rappeler que le bord des ensembles de productions est paramétré par sa projection sur $e^{\perp}$ et que par conséquent c'est dans ce dernier espace que doivent être prises les directions de déplacement.

HYPOTHĖSE $(\mathrm{CN} 2)$ : pour tout équilibre $\left(\left(x_{i}\right),\left(y_{i}\right), p\right)$ de l'économie $\omega$ et pour $j=1, \ldots, n$, pour tout $v \in e^{\perp}$ et $v \neq 0$ on a $v \cdot \frac{d \varphi_{j}}{d y_{j}}\left(y_{j}\right) v \geq 0$.

Bien entendu, cette hypothèse est satisfaite par des ensembles de production convexes, mais il suffit, en fait qu'ils soient localement convexes au voisinage des plans de production d'équilibre pour vérifier cette hypothèse. Comme nous le verrons plus loin, celà pourra notamment être le cas pour des producteurs soumis à une technologie de production à rendements décroissants associée à des coûts fixes de mise en place de cette technologie. En revanche, le contre-exemple de BEATO et MAS-COLELL [1985], où l'un des deux ensembles de production est partout concave et où donc la tarification marginale correspond toujours (pour ce producteur) à une minimisation locale du profit, ne rentre pas dans le cadre de nos hypothèses. Il ne rentrera pas non plus dans le cadre de nos conclusions puisque cet exemple a trois équilibres parfaitement calculables.

Nous imposerons, enfin, l'hypothèse suivante,

HYPOTHÈSE DE TRANSVERSABILITÉ : $\mathrm{Si}\left(\left(x_{i}\right),\left(y_{i}\right), p\right)$ est un équilibre de l'économie $\omega$ et si $\left(v_{1}, \ldots, v_{n}\right) \in\left(e^{\perp}\right)^{n}$ vérifie $\frac{d \varphi_{i}}{d y_{j}}\left(y_{j}\right) v_{j}=0$, $j=1, \ldots, n$ et $v_{1}+\ldots+v_{n}=0$, alors $v_{1}=\ldots=v_{n} \stackrel{\text {. }}{=} 0$.

La signification de cette dernière hypothèse n'est pas immédiate, elle a cependant une interprétation tout à fait naturelle. En effet, si elle n'est pas vérifiée, celà signifie qu'en se déplaçant de façon infinitésimale dans la direction de $\left(v_{1}, \ldots, v_{n}\right)$ à partir de $\left(y_{1}, \ldots, y_{n}\right)$, on reste sur le bord des ensembles de production, le profit des producteurs n'est pas modifié et la production globale est inchangée. Ainsi, malgré une modification de la production, les conditions de l'équilibre restent satisfaites et l'unicité ne peut donc plus être assurée. Remarquons, en outre, que cette hypothèse est trivialement vérifiée dans deux cas particuliers importants:

- si il y a un seul producteur,

- si les producteurs vérifient en fait les conditions suffisantes du second ordre (CS2) de maximisation du profit formalisées ci-après. En effet, dans ce cas, $\frac{d \varphi_{j}}{d y_{j}}\left(y_{j}\right) v_{j}=0$ implique $v_{j}=0$. 
HypothÈSE (CS2) : pour tout équilibre $\left(\left(x_{i}\right),\left(y_{j}\right), p\right)$ de l'économie $\omega$ et pour $j=1, \ldots, n$, pour tout $v \in e^{\perp}$ et $v \neq 0$ on a $v \cdot \frac{d \varphi_{j}}{d y_{j}}\left(y_{j}\right) v>0$.

Nous prouvons, à présent, établir le résultat suivant,

THÉORÈME 3 : Soit $\omega$ une économie « $C^{1}$ » vérifiant les hypothèses (A), $(S(\omega), R(\omega)),(C N 2)$ ainsi que l'hypothèse de transversalité. Supposons de plus que l'économie $\omega$ vérifie soit l'hypothèse de substituabilité brute, soit la loi de la demande généralisée alors l'économie $\omega$ est régulière et admet un unique équilibre.

Corollaire 4 : Sous les hypothèses (A), $(S(\omega)),(R(\omega))$, l'hypothèse de transversabilité et la loi de la demande généralisée (ou l'hypothèse de substituabilité brute), si l'économie régulière $\omega$ admet plus d'un équilibre alors au moins l'un des équilibres correspond à une situation où au moins l'un des producteurs pourrait augmenter son profit dans un voisinage du prix d'équilibre.

Preuve: Grâce au théorème 1 on a, pour les économies régulières,

$$
\sum i_{\left(\left(x_{i}\right),\left(y_{i}\right), p\right)}=(-1)^{\ell-1}
$$

où $i_{\left(\left(x_{i}\right),\left(y_{j}\right), p\right)}=s g \operatorname{det} \Lambda_{0}^{\prime}\left(\left(y_{j}\right), p,\left(\omega_{i}\right)\right)$. De plus, il suffit de démontrer la non nullité de ce dernier déterminant pour tout équilibre de l'économie $\omega$ pour obtenir la régularité de $\omega$. Or, il est facile de voir, par des manipulations élémentaires que

$$
i_{\left(\left(x_{i}\right),\left(y_{j}\right), p\right)}=s g \operatorname{det}\left(\begin{array}{ccccc}
\frac{d \varphi_{1}}{d y_{1}}\left(y_{1}\right) & 0 & \ldots & 0 & -I_{\ell-1} \\
0 & \ddots & \ddots & \vdots & \vdots \\
\vdots & \ddots & \ddots & 0 & \vdots \\
0 & \ldots & 0 & \frac{d \varphi_{n}}{d y_{n}}\left(y_{n}\right) & -I_{\ell-1} \\
\frac{\partial \bar{F}}{\partial y_{1}} & \ldots & \ldots & \frac{\partial \bar{F}}{\partial y_{n}} & \frac{\partial \bar{F}}{\partial \bar{p}}
\end{array}\right)
$$

Soit $A$ cette matrice et remarquons que $\frac{d \varphi_{j}}{d y_{j}}\left(y_{j}\right)$ est la matrice de l'application linéaire tangente à $\varphi_{j}$ et qu'à ce titre elle peut être identifiée à une application de $e^{\perp}$ dans $e^{\perp}$, elle constitue donc une matrice carrée de dimension $(\ell-1)$. Soit $B$ la matrice diagonale par blocs de même dimension que $A$, de blocs diagonaux égaux à l'identité de $e^{\perp}$, à l'exception du dernier égal à $-I d_{e^{\perp}}$. Soit $V=\left(v_{1}, \ldots, v_{n+1}\right)$ un vecteur de $\left(e^{\perp}\right)^{n+1}$. Si l'on démontre que la matrice $B A$ est régulière et que pour tout $V$, $V B A^{t} V$ est positif ou nul on pourra en déduire que le déterminant de $B A$ est strictement positif et par suite que le déterminant de $A$ est du signe de $(-1)^{\ell-1}$. On aura alors, d'une part que l'économie $\omega$ est régulière et d'autre part que l'indice de tout équilibre est égal à $(-1)^{\ell-1}$, ce qui, par la formule de l'indice, impliquera l'unicité de l'équilibre. 
Un calcul élémentaire nous donne

$$
\begin{aligned}
V B A^{t} V= & \sum_{j=1}^{n} v_{j} \frac{d \varphi_{j}}{d y_{j}}{ }^{t} v_{j}-v_{n+1} \sum_{j=1}^{n}{ }^{t} v_{j}-v_{n+1} \\
& \times \sum_{j=1}^{n} \frac{\partial \bar{F}}{\partial y_{j}}{ }^{t} v_{j}-v_{n+1} \frac{\partial \bar{F}}{\partial \bar{p}} v_{n+1} .
\end{aligned}
$$

Or, $\frac{\partial \bar{F}}{\partial \bar{y}}=\sum_{i=1}^{m} \frac{\partial \bar{D}_{i}}{\partial \bar{y}}-I_{\ell-1}$. Or lorsque $y$ varie de façon infinitésimale de manière à rester sur le bord de l'ensemble de production, il est clair que le profit $p \cdot y$ reste inchangé et puisque $D_{i}$ ne dépend de $y$ que par l'intermédiaire de $p \cdot y$ on a $\frac{\partial \bar{D}_{i}}{\partial y}=0$ sur $e^{\perp}$ et $\frac{\partial \bar{F}}{\partial \bar{y}}=-I_{\ell-1}$. De plus, $\frac{\partial \bar{F}}{\partial \bar{p}}=\frac{d \bar{Z}}{d \bar{p}}$. Ainsi,

$$
V B A^{t} V=-v_{n+1} \frac{d \bar{Z}}{d p}{ }^{t} v_{n+1}+\sum_{j=1}^{n} v_{j} \frac{d \varphi_{j}}{d \bar{y}_{j}} t_{j}
$$

D'après la loi de la demande généralisée, pour $v \neq 0$ et $p \cdot v=0,{ }^{t} v \frac{d \bar{Z}}{d \bar{p}} v<0$. De plus, il est facile de vérifier en utilisant la loi de Walras et l'homogénéité de la fonction d'excès de demande que ${ }^{t} p \frac{d \bar{Z}}{d \bar{p}}=\frac{d \bar{Z}}{d \bar{p}} p=0$. Soit alors $v \in e^{\perp}, v$ peut être décomposé sur $p$ et sur $p^{\perp}$ et il est immédiat que cette dernière composante ne peut être nulle. Il est alors facile de voir que pour tout $v \in e^{\perp}, v \neq 0$, on a ${ }^{t} v \frac{d \bar{Z}}{d \bar{p}} v<0$ et le premier terme de la somme précédente est alors strictement positif, dès que $v_{n+1} \neq 0$. Par ailleurs, les autres termes de la somme sont trivialement positifs ou nuls (par l'hypothèse (CN2)), ce qui prouve que $V B A^{t} V$ est toujours positif ou nul et strictement positif si $v_{n+1} \neq 0$. Pour montrer que $B A$ est régulière, il suffit d'étudier son noyau. Or, d'après ce qui précède, il est clair que tout vecteur $V$ du noyau vérifie $v_{n+1}=0$. La condition d'appartenance au noyau devient alors $\frac{d \varphi_{j}}{d y_{j}}\left(y_{j}\right) v_{j}=0, j=1, \ldots, n$ et $v_{1}+\ldots+v_{n}=0$, ce qui par l'hypothèse de transversalité donne $v_{1}=\ldots=v_{n}=0$, puis $V=0$.

Remarquons que le résultat a été démontré sous la loi de la demande généralisée. Mais il est classique de démontrer (voir MAS-CoLELL [1985]) que sous l'hypothèse de substituabilité brute, la loi de la demande généralisée est vérifiée.

Corollaire 5 : Soit $\omega$ une économie " $C^{1}$ » vérifiant les hypothèses (A), $(S(\omega)),(R(\omega))$ et $(C S 2)$. Supposons de plus que l'économie $\omega$ vérifie soit l'hypothèse de substituabilité brute, soit la loi de la demande généralisée alors l'économie $\omega$ est régulière et admet un unique équilibre.

Supposons, à présent que les producteurs disposent de technologies de production à rendements décroissants associées, pour certains d'entre eux, à un coût fixe de mise en place de cette technologie. Nous dirons que l'économie $\omega$ est productive si pour tout équilibre de $\omega$, les producteurs soumis à des coûts fixes sont productifs. On a alors le résultat, 
Corollaire 6 : Soit $\omega$ une économie " $C^{1}$ » vérifiant les hypothèses (A), $(S(\omega)),(R(\omega))$ de transversalité ainsi que la loi de la demande généralisée. Si l'économie $\omega$ est productive alors elle admet un unique équilibre.

De plus, si la partie à rendements décroissants des ensembles de production est différentiellement strictement convexe (i.e. vérifie les conditions suffisantes du second ordre de stricte convexité, (CS2)), l'hypothèse de transversalité peut être omise dans l'énoncé.

Ce résultat est immédiat, puisque si les producteurs sont productifs à l'équilibre, alors ils se situent sur la partie convexe de leur ensemble de production et vérifient, par conséquent, l'hypothèse $(\mathrm{CN} 2)$.

Dans le cas d'un unique ensemble de production, la seule hypothèse restrictive est que l'économie $\omega$ doit être $C^{1}$, c'est-à-dire que, outre la régularité de la demande, l'ensemble de production doit avoir un bord $C^{2}$ au voisinage des plans d'équilibre. Celà est notamment le cas si le secteur productif est défini par une matrice d'activité comme dans SAMUELSON [1951] ou dans KeHOE [1980, 1985], et si pour tout équilibre, $\ell-1$ activités sont opérantes (comme celà est le cas sous des hypothèses vérifiant le théorème de non substitution de Samuelson). En effet, il est facile de voir qu'au voisinage des plans de production d'équilibre, l'ensemble de production est une variété linéaire de dimension $\ell-1$.

Corollaire 7 : Soit une économie $\omega$ dont le secteur productif est défini par une matrice d'activité telle que pour tout équilibre, $\ell-1$ activités sont opérantes, alors sous les hypothèses $(A),(S(\omega)),(R(\omega))$ ainsi que la loi de la demande généralisée, l'économie $\omega$ admet un unique équilibre.

Considérons à présent le processus dynamique suivant, inspiré du tâtonnement de Walras. Pour un prix donné, les producteurs annoncent des plans de production compatibles avec leurs règles de tarification et les consommateurs maximisent alors leur utilité sous la contrainte de budget induite par les prix et les plans de production. Un nouveau prix est alors choisi de telle sorte que, pour un bien donné, le prix augmente si l'offre est inférieure à la demande et diminue sinon.

Afin que les mouvements de prix déterminent parfaitement les mouvements des plans de production, au voisinage de l'équilibre $\varepsilon=$ $\left(\left(x_{i}^{*}\right),\left(y_{i}^{*}\right), p^{*}\right)$, nous allons établir le résultat de stabilité sous la condition suffisante du second ordre de maximisation du profit (CS2). Plus précisément, nous aurons seulement besoin que (CS2) soit vérifiée au voisinage de l'équilibre dont on étudie la stabilité et non pour tous les équilibres de $\omega$. Nous introduisons alors l'hypothèse affaiblie suivante.

HypothèSe $(\operatorname{CS} 2(\varepsilon))$ : pour $j=1, \ldots, n$, pour tout $v \in R^{\ell}, v \neq 0$ et $p^{*} \cdot v=0$ on a $v \cdot \frac{d \varphi_{i}}{d y_{j}}\left(y_{j}^{*}\right) v>0$.

Formellement, soit $\omega$ une économie « $C^{1}$ » vérifiant la condition suffisante du second ordre $(\operatorname{CS} 2(\varepsilon))$ de maximisation du profit en un équilibre $\varepsilon$, l'hypothèse $(\mathrm{CS} 2(\varepsilon))$ implique que les fonctions $\left(\varphi_{j}\right)$ sont régulières et admettent des inverses locales de telle sorte que les plans de production 
sont localement déterminés par les équations $y_{j}=\left(\varphi_{j}^{\varepsilon}\right)^{-1}(p)$. Soit alors le mécanisme défini, dans un voisinage du prix d'équilibre $p^{*}$, par l'équation $\frac{d \bar{p}}{d t}(t)=\bar{z}_{\varepsilon}(p(t))$, où $z_{\varepsilon}(p)$ désigne la valeur de la fonction d'excès de demande calculée pour le prix $p$ et pour les plans de productions $y_{j}=\left(\varphi_{j}^{\varepsilon}\right)^{-1}(p)$.

Proposition 8 : Si l'économie $\omega$ vérifie les hypothèses $(A),(S(\omega))$, $(R(\omega))$ ainsi que la loi de la demande généralisée. Si $\varepsilon$ est un équilibre de l'économie $\omega$ vérifiant l'hypothèse (CS2 $(\varepsilon)$ ), alors $\varepsilon$ est localement asymptotiquement stable pour le processus que nous avons défini et la convergence vers l'équilibre est exponentielle.

Preuve: On a $z_{\varepsilon}(p)=\sum_{i=1}^{m} D_{i}\left(p, p \cdot \omega_{i}+r_{i}\left(p ; p \cdot\left(\varphi_{1}^{\varepsilon}\right)^{-1}(p)\right.\right.$, $\left.\left.\ldots, p \cdot\left(\varphi_{n}^{\varepsilon}\right)^{-1}(p)\right)\right)-\sum_{i=1}^{m} \omega_{i}-\sum_{j=1}^{n}\left(\varphi_{j}^{\varepsilon}\right)^{-1}(p)$ et $\frac{d \bar{z}_{\varepsilon}}{d \bar{p}}=\frac{\partial \bar{F}}{\partial \bar{p}}+$ $\sum_{j=1}^{n} \frac{\partial \bar{F}}{\partial \bar{y}_{j}}\left(\frac{d \varphi_{j}^{e}}{d \bar{y}_{j}}\right)^{-1}$. Il est alors facile de voir, par un calcul analogue à celui utilisé dans la démonstration du théorème 4.1, que pour tout $v \in e^{\perp}$, $v \neq 0,{ }^{t} v \frac{d z_{e}}{d p} v<0$. La stabilité du système dynamique étudié est alors une conséquence classique de la négativité de l'opérateur associé (voir, par exemple, MAS-Colell [1985] ou HiRsch et SMALE [1974]). 
Pour démontrer la formule de l'indice, nous allons procéder par restriction successives. Plus précisément, nous allons montrer que les règles de tarifications, marginales, quoique pouvant engendrer des pertes non bornées, peuvent être remplacées par des règles de tarifications à pertes dites faiblement bornées. Nous montrerons alors que ces dernières peuvent être transformées en règles de tarification satisfaisant une certaine hypothèse de compacité. Après avoir démontré, à chaque étape, que les transformations ne modifient pas le degré de $\Lambda_{0}$, il suffira d'étudier ce degré pour la dernière catégorie de règles de tarifications pour établir le résultat recherché.

Pour celà et dans tout ce qui suit, une règle de tarification générale sera définie par une correspondance $\varphi\left(p,\left(y_{j}\right)\right)$ semi-continue supérieurement à valeurs convexes compactes définie sur $S \times \prod_{j=1}^{n} \partial Y_{j}$ et à valeurs dans $\left(e^{\perp}+\frac{1}{\ell} e\right)^{n}$.

Toutes les correspondances définies précedemment dans le cadre de la règle de tarification marginale restent définies de la même façon (i.e. il suffit de remplacer dans toutes les définitions $\left(\varphi_{j}\left(y_{j}\right)\right)$ par $\left.\varphi\left(p,\left(y_{j}\right)\right)\right)$. De plus, un équilibre de production sera désormais défini par la condition $(p, \ldots, p) \in \varphi\left(p,\left(y_{j}\right)\right)$.

Dans ce qui suit nous allons rappeler, pour une économie $\omega$, quelques hypothèses classiques et résultats importants concernant de telles règles de tarifications.

HYPOTHĖSE FAIBLE DES PERTES BORNÉES : Il existe $t_{0}>0$ tel que pour tout $t \geq t_{0}$, pour tout $\left(p,\left(y_{j}\right)\right) \in S \times \prod_{j=1}^{n} \partial Y_{j}$ et pour tout $\left(q_{j}\right) \in \varphi\left(p,\left(y_{j}\right)\right)$, si $\sum_{i=1}^{m} \omega_{i}+\sum_{j=1}^{n} y_{j}+t e \geq 0$ et $p \cdot\left(\sum_{i=1}^{m} \omega_{i}+\sum_{j=1}^{n} y_{j}+t e\right)=0$ alors $\sum_{j=1}^{n} q_{j} \cdot y_{j} \geq p \cdot \sum_{j=1}^{n} y_{j}$.

HyPOTHÈSE DE COMPACITÉ : Il existe un compact $C$ de $\prod_{j=1}^{n} \partial Y_{j}$ tel que pour tout $\left(p,\left(y_{j}\right)\right) \in S \times \prod_{j=1}^{n} \partial Y_{j}$, si $\varphi\left(p,\left(y_{j}\right)\right) \in S^{n}$ alors $\left(p,\left(y_{j}\right)\right) \in S \times C$.

HYPOTHĖSE FAIBLE DE SURVIVANCE : il existe un sous ensemble connexe $W$ de $R^{m \ell}$ et il existe $\omega_{1} \in R^{m \ell}$ tels que $\omega \in W, \omega_{0}+R_{++}^{m \ell} \subset W$ et pour tout $\omega^{\prime} \in W$ pour tout $\left(p,\left(y_{j}\right)\right) \in S \times \prod_{j=1}^{n} \partial Y_{j}$, si $\left.(p, \ldots, p) \in \varphi\left(p, \varphi_{j}\right)\right)$ et $\sum_{i=1}^{m} \omega_{i}^{\prime}+\sum_{j=1}^{n} y_{j} \geq 0$ alors $p \cdot\left(\sum_{i=1}^{m} \omega_{i}^{\prime}+\sum_{j=1}^{m} y_{j}\right)>0$.

\section{Etape 1: de la tarification marginale aux pertes faiblement bornées}

Le résultat de cette étape est le résultat de la proposition suivante due à BONNISSEAU [1992]. 
Proposition : Sous les hypothèses du théorème 3.2 , il existe une règle de tarification $\varphi$ vérifiant l'hypothèse faible des pertes bornées ainsi que l'hypothèse faible de survivance (avec $\omega_{1}=\omega$ ) telle que pour tout $\left(p,\left(y_{j}\right)\right) \in S \times \prod_{j=1}^{n} \partial Y_{j}$ vérifiant $\sum_{i=1}^{m} \omega_{i}+\sum_{j=1}^{n} y_{j} \geq 0$ on a,

$$
\varphi\left(p,\left(y_{j}\right)\right)=\prod_{j=1}^{n} \varphi_{j}\left(y_{j}\right) .
$$

En d'autres termes, ce résultat permet de remplacer la règle de tarification marginale par une règle de tarification vérifiant une hypothèse faible de pertes bornée ainsi que l'hypothèse faible de survivance, de telle sorte que cette substitution soit neutre sur l'ensemble des allocations admissibles. Il est clair alors, qu'une telle substitution ne modifie ni l'ensemble des équilibres de l'économie $\omega$, ni le degré de la correspondance $\Lambda_{0}$ (cette correspondance étant, à présent, définie, en remplaçant $\prod_{j=1}^{n}\left(\varphi_{j}\left(y_{j}\right)-\{p\}\right)$ $\left.\operatorname{par} \varphi\left(p,\left(y_{j}\right)\right)-\{p, \ldots, p\}\right)$.

Dans toute la suite, $\Lambda_{0}$ désignera cette correspondance modifiée et $\omega$ cette économie modifiée.

\section{Etape 2 : des pertes faiblement bornées à l'hypothèse de compacité}

Soit $t_{0}$ donné par l'hypothèse faible des pertes bornées et quitte à le remplacer par un réel plus grand, on peut supposer $\omega_{0}=\omega+t_{0} e \gg 0$ et soit $K_{Y}=\left\{y=\left(y_{j}\right) \in \prod_{j=1}^{n} \partial Y_{j}: \sum_{j=1}^{n} y_{j}+\sum_{i=1}^{m} \omega_{0, i} \geq 0\right\}$. Pour $y_{j} \in \partial Y_{j}$, on pose $\bar{y}_{j}=\operatorname{proj}_{e^{\perp}}\left(y_{j}\right)$ et $\lambda_{j}\left(\bar{y}_{j}\right)=-\frac{1}{\ell} y_{j} \cdot e$. Il est facile de vérifier que $y_{j}=\bar{y}_{j}-\lambda_{j}\left(\bar{y}_{j}\right) e$. De plus, sous l'hypothèse (A-2), il existe deux réels $A$ et $B$ tels que, pour tout $j$ et tout $y_{j} \in \partial Y_{j}$, on a $\lambda_{j}\left(\bar{y}_{j}\right) \leq A\left\|\bar{y}_{j}\right\|+B$. Soit $f$ la fonction définie sur $\left(e^{\perp}\right)^{n}$, pour $s=\left(s_{j}\right)$, par $f(s)=\sum_{j=1}^{n}\left(\left\|s_{j}\right\|^{2}-A\left\|s_{j}\right\|-B\right)$. Il est immédiat que pour tout réel positif $k$, l'ensemble $\left\{\left(s_{j}\right) \in\left(e^{\perp}\right)^{n}: f(s) \leq k\right\}$ est compact. Soit alors $E \in R^{m \ell}$ défini par $E=(e, \ldots, e)$ et soit $K_{S}$ l'ensemble défini par $K_{S}=\left\{s \in\left(e^{\perp}\right)^{n}: s+\frac{1}{\ell} E \in S^{n}\right\}$. Il est clair que les ensembles $K_{Y}$ et $K_{S}$ sont compacts. Soit alors, $\delta$ un réel positif vérifiant $\delta>\sup _{y \in K_{Y} \text { où } \bar{y} \in K_{S}} f(\bar{y})$.

Définissons, enfin, la fonction $\beta$ sur $\left(e^{\perp}\right)^{n}$ par,

$$
\beta\left(s_{j}\right)=\left\{\begin{array}{l}
0, \quad \text { si } f(s) \leq \delta \\
1, \quad \text { si } f(s) \geq \delta+1 \\
f(s)-\delta, \quad \text { dans tous les autres cas }
\end{array}\right.
$$

et $\psi$, la règle de tarification générale, par

$$
\psi\left(p,\left(y_{j}\right)\right)=\left(1-\beta\left(\left(\bar{y}_{j}\right)\right)\right) \varphi\left(p,\left(y_{j}\right)\right)+\beta\left(\left(\bar{y}_{j}\right)\right)\left(\left(\bar{y}_{j}\right)+\frac{1}{\ell} E\right) .
$$

On peut remarquer que si $\beta\left(\left(y_{j}\right)\right)=1$ alors $\left(\left(\bar{y}_{j}\right)\right) \notin K_{s}$ et $\psi\left(p,\left(y_{j}\right)\right) \notin$ $S^{n}$ et que, par conséquent, si $\psi\left(p,\left(y_{j}\right)\right) \in S^{n}$ alors $f\left(\left(\bar{y}_{j}\right)\right) \leq \delta+1$. L'hypothèse de compacité est alors vérifiée par $\psi$. 
Il est alors assez facile de vérifier que si l'on remplace $\varphi$ par $\psi$ alors l'hypothèse (A) est également vérifiée et que de plus deg $\Lambda_{0}$ n'est pas modifié dans un voisinage de $(0,0, \omega)$.

Vérifions, à présent, que l'hypothèse faible de survivance est également vérifiée.

Soit $\omega^{\prime} \gg \omega_{0}$ et $\left.\left(p, y_{j}\right)\right) \in S \times \prod_{j=1}^{n} \partial Y_{j}$ tels que $\sum_{i=1}^{m} \omega_{i}^{\prime}+\sum_{j=1}^{n} y_{j} \geq 0$ et $(p, \ldots, p) \in \psi\left(p,\left(y_{j}\right)\right)$. Puisque, $p \in S$ on a $\beta\left(\left(y_{j}\right)\right)<1$ et, par conséquent, $f\left(\left(\bar{y}_{j}\right)\right)<\delta+1$ et $\left(\bar{y}_{j}\right)$ est alors borné indépendamment du choix de $\omega^{\prime}$. On en déduit que pour $\omega^{\prime}$ assez grand, on a nécessairement $p \cdot\left(\sum_{i=1}^{m} \omega_{i}^{\prime}+\sum_{j=1}^{n} y_{j}\right)>0$. Il existe alors $t_{1}$ et $\omega_{1}=\omega+t_{1} e$ tels que pour $\omega^{\prime} \geq \omega_{1}$ cette dernière propriété est vérifiée.

Supposons, à présent, que $\omega^{\prime}=\omega+$ te avec $\left.\left.t \in\right] t_{0}, t_{1}\right]$, et soit $\left(p,\left(y_{j}\right)\right) \in$ $S \times \prod_{j=1}^{n} \partial Y_{j}$ tels que $\sum_{i=1}^{m} \omega_{i}^{\prime}+\sum_{j=1}^{n} y_{j} \geq 0, p \cdot\left(\sum_{i=1}^{m} \omega_{i}^{\prime}+\sum_{j=1}^{n} y_{j}\right)=0$ et $(p, \ldots, p) \in \psi\left(p,\left(y_{j}\right)\right)$. De même que précedemment on a $\beta\left(\left(y_{j}\right)\right)<1$ et donc $\frac{1}{1-\beta\left(\left(\bar{y}_{j}\right)\right)}\left(\left(p-\beta\left(\left(\bar{y}_{j}\right)\right)\left(\bar{y}_{j}+\frac{1}{\ell} e\right)\right)\right) \in \varphi\left(p,\left(y_{j}\right)\right)$. L'hypothèse faible des pertes bornées implique alors que,

$$
\sum_{j=1}^{n}\left(p-\beta\left(\left(\bar{y}_{j}\right)\right)\left(\bar{y}_{j}+\frac{1}{\ell} e\right)\right) \cdot y_{j} \geq\left(1-\beta\left(\left(\bar{y}_{j}\right)\right)\right) p \cdot \sum_{j=1}^{n} y_{j}
$$

ce qui implique,

$$
-\beta\left(\left(\bar{y}_{j}\right)\right) \sum_{j=1}^{n}\left(\bar{y}_{j}+\frac{1}{\ell} e\right) \cdot y_{j} \geq-\beta\left(\left(\bar{y}_{j}\right)\right) p \cdot \sum_{j=1}^{n} y_{j},
$$

si l'on suppose de plus que $\beta\left(\left(\bar{y}_{j}\right)\right) \neq 0$ on a alors,

$$
\begin{aligned}
p \cdot \sum_{j=1}^{n} y_{j} & \geq \sum_{j=1}^{n}\left(\bar{y}_{j}+\frac{1}{\ell} e\right) \cdot\left(\bar{y}_{j}-\lambda_{j}\left(\bar{y}_{j}\right) e\right) \\
& \geq \sum_{j=1}^{n}\left(\left\|\bar{y}_{j}\right\|^{2}-A\left\|\bar{y}_{j}\right\|-B\right)>\delta>0
\end{aligned}
$$

et puisque $\omega^{\prime}=\omega+t e \geq \omega_{0} \gg 0$, il vient

$$
p \cdot\left(\sum_{j=1}^{n} y_{j}+\sum_{i=1}^{m} \omega_{i}^{\prime}\right)>0 \text {. }
$$

De même, si $\beta\left(\left(\bar{y}_{j}\right)\right)=0$, on a $\psi\left(p,\left(y_{j}\right)\right)=\varphi\left(p,\left(y_{j}\right)\right)$ et puisque $\varphi$ satisfait l'hypothèse faible de survivance (avec $\omega_{0}=\omega$ ) on a

$$
p\left(\sum_{j=1}^{n} y_{j}+\sum_{i=1}^{m} \omega_{i}^{\prime}\right)>0
$$

Ainsi, dans tous les cas pour $\omega^{\prime} \geq \omega_{1}$ ou $\omega^{\prime}=\omega+$ teavec $\left.\left.t \in\right] t_{0}, t_{1}\right]$, pour tout $\left(p,\left(y_{j}\right)\right) \in S \times \prod_{j=1}^{n} \bar{\partial} Y_{j}$, si $(p, \ldots, p) \in \varphi\left(p,\left(y_{j}\right)\right)$ et $\sum_{i=1}^{m} \omega_{i}^{\prime}+\sum_{j=1}^{n} y_{j} \geq 0$ alors $p \cdot\left(\sum_{i=1}^{m} \omega_{i}^{\prime}+\sum_{j=1}^{n} y_{j}\right)>0$.

Soit à présent $\omega^{\prime} \in R^{m \ell}$ tel que $\omega^{\prime} \gg \omega$ et $\omega^{\prime} \leq \omega_{0}$. Soit $\left(p,\left(y_{j}\right)\right)$ un $\psi$-équilibre de production tel que $\sum_{j=1}^{n} y_{j}+\sum_{i=1}^{m} \omega_{i}^{\prime} \geq 0$, on a $\sum_{j=1}^{n} y_{j}+\sum_{i=1}^{m} \omega_{i}^{*} \geq 0$ et $\left(y_{j}\right) \in K_{Y}$. Par conséquent, $f\left(\left(\bar{y}_{j}\right)\right) \leq \delta$, 
$\beta(s)=0$ et $\psi\left(p,\left(y_{j}\right)\right)=\varphi\left(p,\left(y_{j}\right)\right)$. Puisque $\omega^{\prime} \gg \omega$, l'hypothèse faible de survivance pour $\varphi$ implique que

$$
p \cdot\left(\sum_{j=1}^{n} y_{j}+\sum_{i=1}^{m} \omega_{i}^{\prime}\right)>0
$$

et donc que pour $\omega_{0} \geq \omega^{\prime} \gg \omega$, pour tout $\left(p,\left(y_{j}\right)\right) \in S \times \prod_{j=1}^{n} \partial Y_{j}$, si $(p, \ldots, p) \in \varphi\left(p,\left(y_{j}\right)\right)$ et $\sum_{i=1}^{m} \omega_{i}^{\prime}+\sum_{j=1}^{n} y_{j} \geq 0$ alors $p \cdot\left(\sum_{i=1}^{m} \omega_{i}^{\prime}+\right.$ $\left.\sum_{j=1}^{n} y_{j}\right)>0$.

Ainsi, la propriété précédente est vérifiée sur $\left\{\omega^{\prime} \in R^{m \ell}: \omega \ll \omega^{\prime} \leq \omega_{0}\right\}$ $\left.\left.\cup\left\{\omega^{\prime} \in R^{m \ell}: \omega^{\prime}=\omega_{0}+\left(t-t_{0}\right) e, t \in\right] t_{0}, t_{1}\right]\right\} \cup\left\{\omega^{\prime} \in R^{m \ell}: \omega^{\prime} \geq \omega_{1}\right\}$. Il est facile de vérifier qu'elle est également satisfaite en $\omega$ et en notant $W$ l'ensemble précédent auquel on a adjoint $\omega$, il est clair que $W$ est connexe et que l'hypothèse faible de survivance est vérifiée.

\section{Etape 3 : de l'hypothèse de compacité au degré de $\Lambda_{0}$}

Soit $\Psi: S \times \prod_{j=1}^{n} \partial Y_{j} \rightarrow S^{n+1}$ la correspondance définie par $\left(p,\left(y_{j}\right)\right) \rightarrow\{p\} \times \psi\left(p,\left(y_{j}\right)\right)$.

Proposition : Sous l'hypothèse (A) et l'hypothèse faible de survivance, il existe un voisinage compact $K$ de $(0,0, \omega)$ tel que $\Lambda_{0}^{-1}(K):=$ $\left\{\left(\left(y_{j}\right), p,\left(\omega_{i}\right)\right) \in U: \Lambda_{0}\left(\left(y_{j}\right), p,\left(\omega_{i}\right)\right) \cap K \neq 0\right\}$ est compact. Le degré de $\Lambda_{0}$ est alors constant sur $K$.

Si l'on suppose, en outre l'hypothèse de compacité et l'hypothèse faible de survivance satisfaites, alors on a

$$
\operatorname{deg}\left(\Lambda_{0},(0,0, \omega)\right)=(-1)^{\ell-1} \operatorname{deg} \Psi
$$

Ce résultat est obtenu par un raisonnement totalement analogue à celui de JouINI ([1992], Proposition 8.1 et 8.2). Il suffit alors de remarquer que pour $\left(\left(y_{j}\right)\right)$ assez grand, on a $\psi\left(p,\left(y_{j}\right)\right)=\left(\bar{y}_{j}\right)+\frac{1}{\ell} E$ et donc que $\Psi\left(p,\left(y_{j}\right)\right)=$ $\left(p,\left(\bar{y}_{j}\right)+\frac{1}{\ell} E\right)$, d'où $\operatorname{deg} \Psi=1$, puis $\operatorname{deg}\left(\Lambda_{0},(0,0, \omega)\right)=(-1)^{\ell-1}$.

\section{- Références bibliographiques}

Beato, P., Mas-Colell, A. (1985). - "On the Marginal Cost Pricing Equilibria with Given Tax-subsidy Rules", Journal of Economic Theory, 37, pp. 356-365.

Bonnisseau, J. M. (1992). - "Existence of Equilibria in Presence of Increasing Returns: A Synthesis", Journal of Mathematical Economics, 21, (5), pp. 441-452.

Bonnisseau, J. M., Cornet, B. (1988). - "Existence of Equilibria when Firms Follow Bounded Losses Pricing Rules", Journal of Mathematical Economics, 17, pp. 119-147.

Cellina, A., Lasota, A. (1969). - "A New Approach to the Definition of Topological Degree for Multivalued Mappings", Atti della Academia Nazionale dei Lincei, Rendiconti. Classe de Scienze Fisiche, Mathematiche e Naturali, 47, pp. 434-440.

Clarke, F. (1983). - Optimization and Nonsmooth Analysis. New York: John Wiley. 
Debreu, G. (1959). - Theory of Value. New York: John Wiley.

Debreu, G. (1972). - "Smooth Preferences", Econometrica, 40, pp. 603-615.

Dierker, E. (1982). - "Regular Economies" in Handbook of Mathematical Economics, Vol. 2, edited by K. J. Arrow and M. D. Intriligator, New York, North Holland.

Geanokoplos, J., Shafer, W. (1990). - "Solving Systems of Simultaneous Equations in Economics", Journal of Mathematical Economics, 19, pp. 69-94.

Guesnerie, R. (1975). - "Pareto Optimality in Nonconvex Economies", Econometrica, 43, pp. 1-29.

Hirsch, M. W., Smale, S. (1974). - Differential equations, dynamical systems and linear algebra, Academic Press, New York.

Hurwicz, L., Reiter, S. (1973). - "The Boundedness of the Feasible Set Without Convexity Assumption", International Economic Review, 14, pp. 580-586.

JouinI, E. (1992). - "An Index Theorem for Nonconvex Production Economies", Journal of Economic Theory, 57(1), pp. 176-196.

Jouini, E. (1992). - "Structure de l'ensemble des équilibres d'une économie productive", Annales de l'Institut Henri Poincaré: Analyse non-linéaire, 9(3), pp. 321-336.

KamiYa, K. (1988). - "Existence and Uniqueness of Equilibria with Increasing Returns", Journal of Mathematical Economics, 17, pp. 149-178.

Kenoe, T. (1980). - "An Index Theorem for General Equilibrium Models with Production", Econometrica, 48(5), pp. 1211-1232.

Kenoe, T. (1983). - "Regularity and Index Theory for Economies with Smooth Production Technologies", Econometrica, 51(4), pp. 895-919.

Kenoe, T. (1985). - "Multiplicity of Equilibria and Comparative Statics", Quarterly Journal of Economics, pp. 119-147.

Mas-Colell, A. (1985). - The Theory of General Economic Equilibrium: A Differential Approach. Cambridge: Cambridge University Press.

SAmuelson, P. A. (1951). - "Abstract of a Theorem Concerning Substituability in Open Leontiel Models" in Activity analysis of production and allocation, T. Koopmans, ed. New York, Wiley, pp. 142-146. 